\title{
A REMARK ON THE CONGRUENCE SUBGROUP PROBLEM
}

\author{
J. MENNICKE
}

(dedicated to H.Helling for his 60th birthday)

\begin{abstract}
In the theory of congruence subgroups, one usually shows that, under suitable assumptions, the normal closure of the $m$ th power of an elementary unipotent matrix coincides with the full congruence subgroup $\bmod m$.

For applications, it is sometimes useful to study the subgroup generated by the $m$ th powers of the elementary unipotent elements. We give an elementary proof for the fact that in $\mathrm{SL}_{n}(\mathbf{Z})$ for $n \geq 3$, this subgroup is normal in a suitably defined congruence subgroup of $\mathrm{SL}_{n}(\mathbf{Z})$. Moreover, the two subgroups coincide.
\end{abstract}

During a visit to Bielefeld, Professor A. Janner raised the following question, in the context of an investigation in cristallography:

Consider the two quadratic forms

$$
\begin{aligned}
& f=6 x^{2}-6 x y-3 y^{2}-16 z^{2} \\
& g=-6 x^{2}+6 x y+3 y^{2}-16 z^{2},
\end{aligned}
$$

and the groups of units

$$
U=\mathrm{SO}_{3}(f, \mathrm{Z}), V=\mathrm{SO}_{3}(g, \mathrm{Z}) .
$$

Put $H=\langle U, V\rangle<\mathrm{SL}_{3}(\mathrm{Z})$.

Can one characterise $H$ as a subgroup of $\mathrm{SL}_{3}(\mathrm{Z})$. Is it, in particular, true that

$$
\left|\mathrm{SL}_{3}(\mathrm{Z}): H\right|<\infty ?
$$

I have studied this particular problem. It involves some aspects of the theory of congruence subgroups which are not yet in the literature. Therefore, I present these aspects in this note.

Here are the basic definitions:

Received August 12, 1997. 


$$
\begin{array}{ll}
G=\mathrm{SL}_{n}(\mathrm{Z}), & n \geq 3 \\
m \in \mathrm{N} & \text { subgroup generated by } I+m e_{i j}, \forall 1 \leq i \neq j \leq n . \\
E_{m}=\left\langle I+m e_{i j}\right\rangle, & \left.\begin{array}{l}
a_{i j} \equiv 0 \bmod m \text { for } i \neq j \\
a_{i i}-1 \equiv 0 \bmod m^{2}
\end{array}\right\} \\
G_{m}=\left\{X \in\left(a_{i j}\right) \in G\right.
\end{array}
$$

The standard theory of congruence subgroups proceeds a little differently. One defines

$$
\bar{E}_{m}=N C l_{G}\left(E_{m}\right), \quad \bar{G}_{m}=\left\{X \equiv\left(a_{i j}\right)=\left(\begin{array}{ccc}
1 & & 0 \\
& \ddots & \\
0 & & 1
\end{array}\right) \bmod m\right\},
$$

and one proves $\bar{G}_{m}=\bar{E}_{m}$, involving some number theory (see [1], and more generally [2]).

This is not quite good enough for our purposes, since it may be difficult to obtain full control about a normal closure.

The basic theorems for $E_{m}, G_{m}$ are:

THEOREM 1. $E_{m}=G_{m}$.

One of the main ingredients of the proof of Theorem 1 is

THEOREM 2. $E_{m} \triangleleft G_{m}$.

REMARK. Going through the subsequent proof, it is not difficult to see that Theorem 2 holds much more generally. In fact, a minor modification of our arguments shows that the theorem holds true for Dedekind rings of arithmetical type, in particular for maximal orders in algebraic number fields. Also Theorem 1 generalises. For more details, see [2].

The proofs of Theorem 1 and 2 involve old ideas of L. N. Vaserstein, and of the present author.

Here are some technical tools:

Lemma 1. Let $m \mid m^{\prime}$. Then $G_{m}=G_{m^{\prime}} \cdot E_{m}$.

Proof. Let $X \in G_{m}$ be given. We construct a matrix

$$
\bar{X} \in E_{m}
$$

such that

$$
\begin{aligned}
\bar{X} & \equiv X \bmod m^{\prime} \\
\bar{a}_{i i} & \equiv a_{i i} \bmod m^{\prime 2} .
\end{aligned}
$$

Then

$$
X \cdot \bar{X}^{-1}=Y \in G_{m^{\prime}},
$$


and the Lemma holds.

Put $X=\left(a_{i j}\right) \in G_{m}$.

Put $\left(a_{12}, a_{13}, \ldots, a_{1 n}\right)=c$, the greatest common divisor. Then

$$
\left(c, a_{11}\right)=1 \text {. }
$$

Consider

$$
X\left(\begin{array}{cccc}
1 & & & 0 \\
m t_{2} & 1 & & \\
\vdots & & \ddots & \\
m t_{n} & 0 & & 1
\end{array}\right)=X^{\prime}
$$

We obtain

$$
a_{11}^{\prime}=a_{11}+m\left(t_{2} a_{12}+\cdots+t_{n} a_{1 n}\right) .
$$

$c$ is an integral linear combination of $a_{12}, \ldots, a_{1 n}$, hence we obtain for a suitable choice of $t_{2}, \ldots, t_{n}$ :

$$
a_{11}^{\prime}=a_{11}+m t c .
$$

Because of $\left(a_{11}, c\right)=1$ and $\left(a_{11}, m\right)=1$, we can use the Dirichlet Theorem on primes in arithmetic progressions to produce

$$
a_{11}^{\prime}=p, a \text { prime, and }\left(p, m^{\prime}\right)=1,\left(p, a_{1 n}\right)=1 .
$$

Hence we may assume from the beginning $a_{11}=p$, and (1) holds.

Consider

$$
X \cdot\left(I+t m e_{1 n}\right)=\left(\begin{array}{llll}
a_{11} & a_{12} & \ldots & a_{1 n}+t m a_{11} \\
& & * * &
\end{array}\right)
$$

Put $a_{1 n}=m a_{1 n}^{*}$, obtaining

$$
a_{1 n}^{\prime}=m\left(a_{1 n}^{*}+t a_{11}\right)
$$

Because of $\left(a_{11}, a_{1 n}\right)=1$, we can invoke Dirichlet's Theorem, obtaining

$$
a_{1 n}^{\prime}=m q,
$$

$q$ a prime number, $\left(q, m^{\prime}\right)=1$.

Consider $X \cdot\left(I+t m e_{n 1}\right)$, obtaining

$$
a_{11}^{\prime}=a_{11}+m t a_{1 n}^{\prime}=a_{11}+m^{2} t q .
$$

Solve the congruence

$$
a_{11}^{\prime} \equiv 1 \bmod m^{\prime 2}
$$


Let $m^{\prime}=m d$. Because of $a_{11}=1+m^{2} a_{11}^{*}$, we have $a_{11}^{\prime}=1+m^{2} a_{11}^{*}+m^{2} t q$, hence the congruence (2) reads

$$
a_{11}^{*}+t q \equiv 0 \bmod d^{2} .
$$

Because of $\left(q, m^{\prime}\right)=1$, we have $(q, d)=1$, and hence the congruence (2) can be solved.

For $2 \leq j \leq n$, consider $X\left(I+\right.$ tme $\left._{1 j}\right)$, obtaining

$$
a_{1 j}^{\prime}=a_{1 j}+t m a_{11} .
$$

One can solve the congruence $a_{1 j}+t m a_{11} \equiv 0 \bmod m^{\prime}$, obtaining

$$
\begin{gathered}
X=\left(a_{i j}\right), \\
a_{11} \equiv 1 \bmod m^{\prime 2}, a_{1 j} \equiv 0 \bmod m^{\prime}, 2 \leq j \leq n
\end{gathered}
$$

Consider for $2 \leq j \leq n:\left(I+t m e_{j 1}\right) \cdot X$, obtaining

$$
a_{j 1}^{\prime}=a_{j 1}+t m a_{11}
$$

One can solve the congruence $a_{j 1}+t m a_{11} \equiv 0 \bmod m^{\prime}, 2 \leq j \leq n$, obtaining

$$
\begin{gathered}
X=\left(a_{i j}\right) \\
a_{11} \equiv 1 \bmod m^{\prime 2} \\
a_{1 j} \equiv a_{j 1} \equiv 0 \bmod m^{\prime}, \quad 2 \leq j \leq n .
\end{gathered}
$$

Repeat the argument, obtaining

$$
\begin{gathered}
X=\left(a_{i j}\right) \\
a_{i i} \equiv 1 \bmod m^{\prime 2} \\
a_{i j} \equiv a_{j i} \equiv 0 \bmod m^{\prime}, i \neq j 1 \leq i, j \leq n,
\end{gathered}
$$

and hence we have for the originally given $X \in G_{n}$ :

$$
\begin{gathered}
E_{1} \ldots E_{k} \cdot X \cdot E_{k+1} \cdots E_{r} \in G_{m^{\prime}}, \\
E_{j} \in E_{m} .
\end{gathered}
$$

Put $\quad E_{k}^{-1} \ldots E_{1}^{-1} \cdot E_{r}^{-1} \ldots E_{k+1}^{-1}=\bar{X} \in E_{m}, \quad$ obtaining $\quad \bar{X} \equiv X \bmod m^{\prime}, \bar{a}_{i i} \equiv$ $a_{i i} \bmod m^{\prime 2}$, and hence

$$
X \cdot \bar{X}^{-1}=Y \in G_{m^{\prime}} .
$$

The proof of Lemma 1 is complete.

The cornerstone of the argument is

LemMA 2. Let $m \neq 0$ and $g \in \mathrm{SL}_{n}(\mathrm{Q})$ be given and assume $n \geq 3$.

$\Rightarrow$ There exists an $m^{\prime} \neq 0$ such that

$$
X^{-1} g X g^{-1} \in E_{m} \text { for all } X \in G_{m^{\prime}} .
$$


Historical Remark. An analogue of Lemma 2, for the group $\mathrm{SL}_{2}$ over maximal orders in algebraic number fields with infinitely many units, was first proved by L. N. Vaserstein (see [3]).

Our Lemma 2 is a modification of Vaserstein's Lemma. The ring $\mathbf{Z}$ has only the units \pm 1 . In order to balance this deficiency, we work in $\mathrm{SL}_{n}$ for $n \geq 3$. For $\mathrm{SL}_{2}(\mathrm{Z})$ and $\mathrm{Q}$, the Lemma 2 does not hold.

We show how Lemma 2 implies Theorem 2.

Proof of Theorem 2. Let $X \in G_{m}$ be given, and choose $g=I+m e_{i j}, i \neq j$. For these given data $m, g$, Lemma 2 gives the existence of $m^{\prime} \neq 0$ such that

$$
Y^{-1} g Y^{-1} \in E_{m} \text { for all } Y \in G_{m^{\prime}} .
$$

Let $\left(m, m^{\prime}\right)=d$. Then $m^{\prime \prime}=\frac{m m^{\prime}}{d}=\left[m, m^{\prime}\right]$ is the smallest common multiple of $m, m^{\prime}$.

Choose $Y \in G_{m^{\prime}}$ such that it satisfies further congruences:

$$
Y \in G_{m^{\prime \prime}} .
$$

Then $m \mid m^{\prime \prime}$, and one can use Lemma 1 to find $E \in E_{m}$ such that

$$
Y=X E, \quad E \in E_{m} .
$$

We obtain

$$
\begin{gathered}
E^{-1} \cdot X^{-1} \cdot g \cdot X E \cdot g^{-1} \in E_{m}, \quad \text { and hence } \\
X^{-1} g X \in E_{m} .
\end{gathered}
$$

Since this holds for all $X \in G_{m}$, we have proved Theorem 2, modulo Lemma 2.

The proof of Lemma 2 will be broken up into several steps.

Lemma 3. Let $m \neq 0$ and $g \in \mathrm{GL}_{n}(\mathrm{O})$ be given.

$\Rightarrow$ There exists $m^{\prime \prime} \neq 0$ such that

$$
E_{m^{\prime \prime}}<g E_{m} g^{-1} .
$$

Proof. We distinguish several cases:

Case 1. Consider

$$
g=\left(\begin{array}{rrr}
x_{1} & & 0 \\
& x_{2} & \\
& \ddots & \\
0 & & x_{n}
\end{array}\right), x_{i}=\frac{u_{i}}{v_{i}}, u_{i}, v_{i} \in \mathbf{Z} .
$$

We have: 


$$
g \cdot\left(I+m e_{i j}\right) g^{-1}=I+m x_{i} x_{j}^{-1} e_{i j} .
$$

Choose

$$
m^{\prime \prime}=m u_{1} \ldots u_{n} v_{1} \ldots v_{n} .
$$

Let

$$
N=u_{j}^{2} v_{i}^{2} \prod_{l \neq i, j} u_{l} \cdot \prod_{l \neq i, j} v_{l}
$$

We obtain

$$
\left(I+m x_{i} x_{j}^{-1} e_{i j}\right)^{N}=I+m^{\prime \prime} e_{i j}
$$

Hence we have

$$
\begin{gathered}
I+m^{\prime \prime} e_{i j}<g \cdot E_{m} \cdot g^{-1} \text { for all } i \neq j, \text { and hence } \\
E_{m^{\prime \prime}}<g E_{m} g^{-1} .
\end{gathered}
$$

Case 2. Consider

$$
g=I+e_{12}=\left(\begin{array}{cccc}
1 & 1 & & 0 \\
& 1 & & \\
& & \ddots & \\
0 & & & 1
\end{array}\right)
$$

For $i, j \neq 1,2$, the elements $g$ and $I+m e_{i j}$ commute:

$$
\begin{gathered}
I+m e_{i j} \rightleftarrows g, \quad \text { and hence } \\
I+m e_{i j} \in g E_{m} g^{-1}, \quad i, j \neq 1,2
\end{gathered}
$$

Also, $I+m e_{1 j} \rightleftarrows g$ and hence

$$
I+m e_{1 j} \in g E_{m} g^{-1}
$$

For $j>2$, we have

$$
\begin{gathered}
g^{-1}\left(I+m e_{2 j}\right) g=\left(I+m e_{2 j}\right)\left(I-m e_{1 j}\right), \text { and hence } \\
I+m e_{2 j} \in g E_{m} g^{-1} \text { for } j>2 .
\end{gathered}
$$

We have

$$
g^{-1}\left(I+m e_{j 1}\right) g=\left(I+m e_{j 1}\right)\left(I+m e_{j 2}\right) \text { for } j>2,
$$

and hence

$$
I+m e_{j 1} \in g E_{m} g^{-1} \quad \text { for } j>2 .
$$

We have 


$$
\begin{gathered}
I+m e_{j 2} \rightleftarrows g, \text { and hence } \\
I+m e_{j 2} \in g E_{m} g^{-1} .
\end{gathered}
$$

Hence it remains to consider $I+k e_{21}$.

We have

$$
I+m^{2} e_{21}=\left(I+m e_{23}\right)\left(I+m e_{31}\right)\left(I-m e_{23}\right)\left(I-m e_{31}\right)
$$

This implies:

$$
\begin{aligned}
I+m^{2} e_{21}= & g \cdot g^{-1}\left(I+m e_{23}\right) g \cdot g^{-1}\left(I+m e_{31}\right) g \\
& \cdot g^{-1}\left(I-m e_{23}\right) g \cdot g^{-1}\left(I-m e_{31}\right) g \cdot g^{-1} \\
= & g \cdot\left(I-m e_{13}\right)\left(I+m e_{23}\right)\left(I+m e_{31}\right)\left(I+m e_{32}\right)\left(I+m e_{13}\right) \\
& \cdot\left(I-m e_{23}\right)\left(I-m e_{31}\right)\left(I+m e_{32}\right) \cdot g^{-1} .
\end{aligned}
$$

Hence we have

$$
E_{m^{\prime \prime}}<g E_{m} g^{-1} \text { for } m^{\prime \prime}=m^{2} .
$$

Case 3. Let $g$ be a permutation matrix, i.e. a matrix which has precisely one entry +1 in each row and column, and 0 otherwise. Because of

$$
\begin{gathered}
g\left(I+m e_{i j}\right) g^{-1}=I+m e_{k l}, \quad \text { we have } \\
E_{m^{\prime \prime}}<g E_{m} g^{-1} \quad \text { for } m^{\prime \prime}=m .
\end{gathered}
$$

Case 4. An arbitrary element $g \in \mathrm{GL}_{n}(\mathrm{Q})$ is a product of matrices of types 1), 2), 3). Apply repeatedly the arguments given under 1), 2), 3), completing the proof of Lemma 3.

Lemma 4. The elements $g \in \mathrm{GL}_{n}(\mathrm{O})$ for which Lemma 2 holds true form a subgroup $W<\mathrm{GL}_{n}(\mathrm{Q})$. $W$ is even a normal subgroup in $\mathrm{GL}_{n}(\mathrm{Q})$.

$$
W \triangleleft \mathrm{GL}_{n}(\mathrm{Q}) \text {. }
$$

Proof. The elements $g \in \mathrm{GL}_{n}(\mathrm{Q})$ for which Lemma 2 holds true are precisely those elements for which there is a function

$$
m \rightarrow m^{\prime} \text { for all } m \in \mathrm{N}, m^{\prime} \neq 0
$$

such that $X^{-1} g X g^{-1} \in E_{m}$ for all $X \in G_{m^{\prime}}$.

Let $g, g_{1}$ be two elements for which Lemma 2 holds true. For $g, m$, use Lemma 3 to determine $m^{\prime \prime}$ such that

$$
E_{m^{\prime \prime}}<g E_{m} g^{-1}
$$

Because Lemma 2 holds true for $g, g_{1}$ start out from $m^{\prime \prime}$, and determine $m^{*}, m^{* *}$ for $m^{\prime \prime}$ and $g, g_{1}$, respectively such that 


$$
\begin{aligned}
X^{-1} g X^{-1} \in E_{m^{\prime \prime}} & \text { for all } X \in G_{m^{*}} \\
Y^{-1} \cdot g_{1} Y \cdot g_{1}^{-1} \in E_{m^{\prime \prime}} & \text { for all } Y \in G_{m^{* *}} .
\end{aligned}
$$

Conclude for $m^{+}=m^{*} \cdot m^{* *}$ :

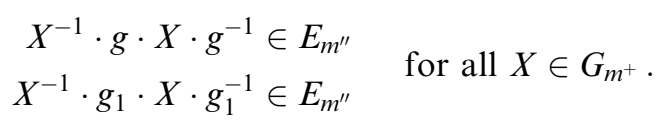

This implies

$$
\begin{aligned}
g \cdot X^{-1} \cdot g^{-1} X \cdot X^{-1} g_{1} X g_{1}^{-1} \in E_{m^{\prime \prime}}, & \text { and hence } \\
g \cdot X^{-1} \cdot g^{-1} g_{1} X \cdot g_{1}^{-1} \in E_{m^{\prime \prime}} & \text { for all } X \in G_{m^{+}} .
\end{aligned}
$$

This implies

$$
X^{-1} \cdot g^{-1} g_{1} \cdot X \cdot g_{1}^{-1} g \in g^{-1} E_{m^{\prime \prime}} g<E_{m} \text { for all } X \in G_{m^{+}} .
$$

Hence we have constructed a function

$$
m \longrightarrow m^{+}
$$

for the element $g^{-1} g_{1}$. Hence Lemma 2 holds for $g^{-1} g_{1}$. Hence the elements for which Lemma 2 holds true form a subgroup

$$
W<\mathrm{GL}_{n}(\mathrm{Q}) \text {. }
$$

Next we prove that the subgroup $W$ is normal in $\mathrm{GL}_{n}(\mathrm{Q})$.

Let $g \in W$ and $g_{1} \in \mathrm{GL}_{n}(\mathbf{Q})$.

Use Lemma 3 to determine for $g_{1}^{-1}$ and $m$ an element $m^{\prime \prime} \neq 0$ such that

$$
E_{m^{\prime \prime}}<g_{1}^{-1} E_{m} g_{1} .
$$

For $m^{\prime \prime}, g$, determine $m^{*}$ such that

$$
X^{-1} g X g^{-1} \in E_{m^{\prime \prime}} \quad \text { for all } X \in G_{m^{*}} .
$$

Hence we obtain

$$
g_{1} \cdot X^{-1} g X g^{-1} g_{1}^{-1} \in g_{1} E_{m^{\prime \prime}} g_{1}^{-1}<E_{m} \quad \text { for all } X \in G_{m^{*}} .
$$

Rewrite this equation obtaining

$$
\left(g_{1} X g_{1}^{-1}\right)^{-1} \cdot g_{1} g g_{1}^{-1}\left(g_{1} X g_{1}^{-1}\right) \cdot\left(g_{1} g g_{1}^{-1}\right)^{-1} \in E_{m} \text { for all } X \in G_{m^{*}} .
$$

Put $Y:=g_{1} X g_{1}^{-1}, X=g_{1}^{-1} Y g_{1}$. Because $g_{1} \in \mathrm{GL}_{n}(\mathrm{Q})$, there is $N \in \mathrm{N}$ such that

$$
g_{1}=\frac{1}{N} g_{2}, g_{2} \in M_{n}(\mathbf{Z}), \operatorname{det} g_{2}=M \in \mathbf{Z}
$$


Choose $M \in \mathrm{N}$ minimal with this property. We obtain

$$
\begin{array}{ll}
g_{2}=N g_{1}, g_{2}^{-1}=N^{-1} g_{1}^{-1}, & \text { and hence } \\
g_{1}^{-1} Y g_{1}=N \cdot g_{2}^{-1} Y \frac{1}{N} g_{2}=g_{2}^{-1} Y g_{2} . &
\end{array}
$$

The inverse of $g_{2}$ :

$$
g_{2}^{-1}=\frac{1}{M} g_{3}, g_{3} \in M_{n}(\mathrm{Z})
$$

Hence we have

$$
g_{3} \cdot g_{2}=M \cdot I
$$

Choose $M^{\prime}=M m^{* 2}$, obtaining for $Y \in G_{M^{\prime}}$ :

$$
g_{1}^{-1} Y g_{1}=g_{2}^{-1} Y g_{2}=\frac{1}{M} g_{3} Y g_{2} .
$$

The matrix $g_{3} Y_{2} \in M_{n}(\mathrm{Z})$ has integral entries, and we can compute congruences $\bmod M^{\prime}, M^{\prime}=M m^{* 2}$, obtaining

$$
M g_{1}^{-1} Y g_{1} \equiv g_{3} Y g_{2} \equiv g_{3} \cdot I g_{2} \equiv g_{3} g_{2} \equiv M I \bmod M m^{* 2} \text {. }
$$

Dividing all entries by $M$, we find

$$
g_{1}^{-1} Y g_{1} \in M_{n}(\mathbf{Z}),
$$

and moreover: $g_{1}^{-1} Y g_{1} \equiv I \bmod m^{* 2}$.

Hence we have, in particular

$$
g_{1}^{-1} Y g_{1}=X \in G_{m^{*}} \text { for all } Y \in G_{M^{\prime}} .
$$

Hence we conclude

$$
Y^{-1} \cdot g_{1} g g_{1}^{-1} \cdot Y \cdot\left(g_{1} g g_{1}^{-1}\right)^{-1} \in E_{m} \text { for all } Y \in G_{M^{\prime}} .
$$

Hence we have constructed a function

$$
m \longrightarrow M^{\prime}
$$

such that

$$
Y^{-1} \cdot g_{1} g g_{1}^{-1} \cdot Y \cdot\left(g_{1} g g_{1}^{-1}\right)^{-1} \in E_{m} \text { for all } Y \in G_{M^{\prime}} .
$$

Hence $g_{1} g g_{1}^{-1} \in W$ for all $g \in W$ and all $g_{1} \in \mathrm{GL}_{n}(\mathrm{Q})$.

Hence $W \triangleleft \mathrm{GL}_{n}(\mathrm{Q})$ is a normal subgroup.

We have completed the proof of Lemma 4.

Remember that the group $\mathrm{GL}_{n}(\mathrm{Q})$ has no normal non-central subgroups 
not containing $\mathrm{SL}_{n}(\mathrm{Q})$. Hence in order to prove Lemma 2, it suffices to produce a non-central element $g \in \mathrm{SL}_{n}(\mathrm{Q})$ for which Lemma 2 holds true.

LeMma 5. For

$$
g=\left(\begin{array}{cccccc}
b & & & & & 0 \\
& b & & & & \\
& & b^{-2} & & & \\
& & & 1 & & \\
& & & & \ddots & \\
0 & & & & & 1
\end{array}\right), \begin{aligned}
& b \in \mathrm{N}, \\
& b^{2} \neq 1,
\end{aligned}
$$

and for the function

$$
m \longrightarrow m^{\prime}=b^{3} m,
$$

Lemma 2 holds true.

Proof. Let $X \in G_{m^{\prime}}, X=\left(a_{i j}\right)$. Consider

Proof.

$$
X\left(\begin{array}{ccccc}
1 & & & \\
0 & 1 & & 0 & \\
t_{3} m^{\prime} & & 1 & & \\
\vdots & & \ddots & \\
t_{n} m^{\prime} & & & & 1
\end{array}\right)=\left(\begin{array}{cccc}
* & & & \\
& & & \\
& & & \\
a_{n 1}^{\prime} & a_{n 2} & \ldots & a_{n n}
\end{array}\right)
$$

obtaining

$$
a_{n 1}^{\prime}=a_{n 1}+t_{3} m^{\prime} a_{n 3}+\cdots+t_{n} m^{\prime} a_{n n}
$$

Put $a_{n 1}=m^{\prime} a_{n 1}^{*}$

$$
d=\left(a_{n 3}, \ldots, a_{n n}\right), \quad a_{n j}=d a_{n j}^{\prime}, \quad 3 \leq j \leq n,
$$

obtaining

$$
a_{n 1}^{\prime}=m^{\prime} a_{n 1}^{*}+m^{\prime} d\left(t_{3} a_{n 3}^{\prime}+\cdots+t_{n} a_{n n}^{\prime}\right) .
$$

If $p \mid d$ and $p \mid a_{n 2}$, then $p \nmid m^{\prime}$ and $p \nmid a_{n 1}$, hence $p \nmid a_{n 1}^{*}$. If $p \mid d$ and $p \mid a_{n 1}$, then $p \nmid m^{\prime}$ and $p \nmid a_{n 2}$.

Choose $t_{3}, \ldots t_{n}$ such that

$$
\begin{array}{cl}
t_{3} a_{n 3}^{\prime}+\cdots+t_{n} a_{n n}^{\prime}=1 & \text { for } n \geq 4, \text { and } \\
a_{n 1}^{\prime}=m^{\prime}\left(a_{n 1}^{*}+t a_{33}^{\prime}\right) & \text { for } n=3, \text { such that } \\
\left(a_{31}^{\prime}, a_{32}\right)=m^{\prime} . &
\end{array}
$$

In both cases we have 


$$
\left(a_{n 1}^{\prime}, a_{n 2}\right)=m^{\prime}
$$

Consider

$$
X \cdot\left(I+t m^{\prime} e_{1 n}+r m^{\prime} e_{2 n}\right)=\left(\begin{array}{cccc}
* & & & \\
a_{n 1} & \cdots & a_{n n-1} & a_{n n}^{\prime}
\end{array}\right),
$$

obtaining

$$
a_{n n}^{\prime}=a_{n n}+t m^{\prime} a_{n 1}+r m^{\prime} a_{n 2} .
$$

From $a_{n 1}=m^{\prime} a_{n 1}^{\prime}, a_{n 2}=m^{\prime} a_{n 2}^{\prime},\left(a_{n 1}^{\prime}, a_{n 2}^{\prime}\right)=1$ we conclude

$$
a_{n n}^{\prime}=a_{n n}+m^{\prime 2}\left(t a_{n 1}^{\prime}+r a_{n 2}^{\prime}\right) \text {. }
$$

Because of $a_{n n} \equiv 1 \bmod m^{\prime 2}$ we can choose $t, r \in \mathrm{Z}$ such that

$$
a_{n n}^{\prime}=1 \text {. }
$$

Consider

$X\left(\begin{array}{ccccc}1 & & & & \\ & 1 & & \\ & & \ddots & & \\ & & & 1 & \\ t_{1} m^{\prime} & t_{2} m^{\prime} & \ldots & t_{n-1} m^{\prime} & 1\end{array}\right)=\left(\begin{array}{ccc}* & & \\ & & \\ a_{n 1}+t_{1} m^{\prime} & a_{n 2}+t_{2} m^{\prime} \ldots a_{n n-1}+t_{n-1} m^{\prime} & 1\end{array}\right)$.

Choose $t_{i} \in \mathrm{Z}$ such that

$$
X=\left(\begin{array}{ccccc}
* & & & & \\
0 & 0 & \ldots & 0 & 1
\end{array}\right) .
$$

Consider

$$
\left(\begin{array}{ccccc}
1 & & & & m^{\prime} u_{1} \\
& 1 & & & m^{\prime} u_{2} \\
& & \ddots & & \vdots \\
& & & 1 & m^{\prime} u_{n-1} \\
& & & & 1
\end{array}\right) X=\left(\begin{array}{ccccc}
* & & & 0 \\
& & & 0 \\
& & & \vdots \\
& & & \\
& & & &
\end{array}\right.
$$

Define

$$
\begin{gathered}
K_{n}=\left\langle I+m^{\prime} e_{n j}, j=1, \ldots, n-1\right. \\
I+m^{\prime} e_{j 1}, j=3, \ldots, n \\
\left.I+m^{\prime} e_{1 n}, I+m^{\prime} e_{2 n}\right\rangle \\
H_{n}=\left\langle I+m^{\prime} e_{j n}, j=1, \ldots, n-1\right\rangle .
\end{gathered}
$$

We have shown that 


$$
\begin{aligned}
& X=E_{n} \cdot X_{n-1} \cdot F_{n}, \\
& E_{n} \in H_{n}, F_{n} \in K_{n} .
\end{aligned}
$$

Define

$$
\begin{aligned}
K_{n-i}= & \left\langle I+m^{\prime} e_{n-i, j}, j=1, \ldots, n-i-1\right. \\
& I+m^{\prime} e_{j 1}, j=3, \ldots, n-i \\
& \left.I+m^{\prime} e_{1 n-i}, I+m^{\prime} e_{2, n-i}\right\rangle \\
H_{n-i}= & \left\langle I+m^{\prime} e_{j, n-i}, j=1, \ldots, n-i-1\right\rangle,
\end{aligned}
$$

for $i=0,1,2, \ldots, n-3$.

The above argument yields a decomposition

$$
\begin{gathered}
X=E_{n} E_{n-1} \ldots E_{3} \cdot Y \cdot F_{3} \ldots F_{n-1} F_{n}, \\
Y=\left(\begin{array}{lllll}
b_{11} & b_{12} & & & 0 \\
b_{21} & b_{22} & & & \\
& & 1 & & \\
& & & \ddots & \\
0 & & & & 1
\end{array}\right) \\
E_{n-i} \in H_{n-i} i=0,1, \ldots, n-3 . \\
F_{n-i} \in K_{n-i}
\end{gathered}
$$

The assertion of Lemma 2 can be written this way:

$$
F_{n}^{-1} \ldots F_{3}^{-1} Y^{-1} E_{3}^{-1} \ldots E_{n}^{-1} g \cdot E_{n} \ldots E_{3} Y F_{3} \ldots F_{n} g^{-1} \in E_{m} .
$$

Because of $m \mid m^{\prime}$, the assertion reduces to

$$
Y^{-1} E_{3}^{-1} \ldots E_{n}^{-1} \cdot g \cdot E_{n} \ldots E_{3} \cdot Y \cdot F_{3} \ldots F_{n} g^{-1} \in E_{m} .
$$

We have

$$
\begin{array}{lll}
I+m^{\prime} e_{n j} \rightleftarrows g & \text { for } j \geq 4 \\
g\left(I+m^{\prime} t e_{n 1}\right) g^{-1} & =I+b^{-1} m^{\prime} t e_{n 1} & \\
g\left(I+m^{\prime} t e_{n 2}\right) g^{-1} & =I+b^{-1} m^{\prime} t e_{n 2} & \text { for } n \geq 4 \\
g\left(I+m^{\prime} t e_{n 3}\right) g^{-1} & =I+m^{\prime} t b^{2} e_{n 3} & \\
g\left(I+m^{\prime} t e_{31}\right) g^{-1} & =I+b^{-3} m^{\prime} t e_{31} & \\
g\left(I+m^{\prime} t e_{32}\right) g^{-1} & =I+b^{-3} m^{\prime} t e_{32} & \\
g\left(I+m^{\prime} t e_{j 1}\right) g^{-1} & =I+b^{-1} m^{\prime} t e_{j 1} & \text { for } j>3 \\
g\left(I+m^{\prime} t e_{1 n}\right) g^{-1} & =I+b m^{\prime} t e_{1 n} & \\
g\left(I+m^{\prime} t e_{2 n}\right) g^{-1} & =I+b m^{\prime} t e_{2 n} & \text { for } n \geq 4 \\
g\left(I+m^{\prime} t e_{13}\right) g^{-1} & =I+m^{\prime} b^{3} t e_{13} & \\
g\left(I+m^{\prime} t e_{23}\right) g^{-1} & =I+m^{\prime} b^{3} t e_{23} . &
\end{array}
$$


Hence one can shift the elements of $K_{n}$ across $g$, reducing the assertion to

$$
Y^{-1} \cdot E_{3}^{-1} \ldots E_{n}^{-1} \cdot g \cdot E_{n} \ldots E_{3} \cdot Y \cdot F_{3} \ldots F_{n-1} g^{-1} \in E_{m} .
$$

For the elements of $K_{n-i}$, one needs the formulas

$$
\begin{aligned}
& I+m^{\prime} e_{\mu, \nu} \rightleftarrows g \quad \text { for } \mu, \nu \geq 4 \\
& g\left(I+m^{\prime} t e_{n-i, 1}\right) g^{-1}=I+m^{\prime} t b^{-1} e_{n-i, 1} \\
& g\left(I+m^{\prime} t e_{n-i, 2}\right) g^{-1}=I+m^{\prime} t b^{-1} e_{n-i, 2} \quad \text { for } n-i \geq 4 \\
& g\left(I+m^{\prime} t e_{n-i, 3}\right) g^{-1}=I+m^{\prime} t b^{2} e_{n-i, 3} \\
& g\left(I+m^{\prime} t e_{j 1}\right) g^{-1}=I+m^{\prime} t b^{-1} e_{j 1} \\
& g\left(I+m^{\prime} t e_{1 n-i}\right) g^{-1}=I+b m^{\prime} t e_{1 n-i} \quad \text { for } n-i \geq 4 \\
& g\left(I+m^{\prime} t e_{2, n-i}\right) g^{-1}=I+b m^{\prime} t e_{2 n-i}
\end{aligned}
$$

These formulas permit to shift the factors $F_{3}, \ldots, F_{n}$ across $g$, reducing the assertion to

$$
Y^{-1} E_{3}^{-1} \ldots E_{n}^{-1} g E_{n} \ldots E_{3} Y g^{-1} \in E_{m} .
$$

The elements $Y, g$ commute:

$$
Y \rightleftarrows g
$$

reducing the assertion to

$$
Y^{-1} \cdot E_{3}^{-1} \ldots E_{n}^{-1} g E_{n} \ldots E_{3} \cdot g^{-1} \cdot Y \in E_{m}
$$

We have

$$
\begin{array}{lll}
I+m^{\prime} e_{j, n-i} & \rightleftarrows g & \text { for } j \geq 4, n-i \geq 4 \\
I+m^{\prime} e_{j, n-i} \rightleftarrows Y & \text { for } j \geq 3, n-i \geq 3
\end{array}
$$

For $n-i=3$ and $j=1,2$, we have

$$
g\left(I+m^{\prime} t e_{j 3}\right) g^{-1}=I+m^{\prime} b^{3} t e_{j 3} .
$$

Hence one can shift all elements $E_{n-i}$ across $g$, possibly changing exponents. For $n-i=3, j=1,2$ we use the fact that these elements are normalized by $Y$.

The same argument holds for $j=1,2$ and $n-i \geq 4$.

Hence we have proved that for 


$$
g=\left(\begin{array}{rrrrr}
b & & & 0 & \\
& b & & & \\
& & b^{-2} & & \\
& & 1 & & \\
& & & \ddots & \\
0 & & & & 1
\end{array}\right), b \in \mathrm{N}, b^{2} \neq 1
$$

and $m^{\prime}=b^{3} m$ the Lemma 2 holds true. We have completed the proof of Lemma 5. We have also completed the proof of Theorem 2:

$$
E_{m} \triangleleft G_{m} .
$$

Lemma 6. For

$$
X=\left(\begin{array}{ccc}
a & b & a \\
c & d & a \\
0 & 0 & 1
\end{array}\right) \in G_{m}, \quad Y=\left(\begin{array}{ccc}
a & b^{\prime} & 0 \\
c^{\prime} & d^{\prime} & 0 \\
0 & 0 & 1
\end{array}\right) \in G_{m},
$$

we have

$$
X \cdot Y \equiv\left(\begin{array}{ccc}
a & b b^{\prime} & 0 \\
c^{\prime \prime} & d^{\prime \prime} & 0 \\
0 & 0 & 1
\end{array}\right) \bmod E_{m} .
$$

The Lemma holds in much greater generality, and is a consequence of known facts, see in particular Lemmas 8 and 10 of [6].

Lemma 6 obviously implies Theorem 1, see [1].

We have established Theorem 1.

We now come to the question posed by Professor Janner.

It is a routine matter to compute the groups of units of the forms $f$ and $g$, respectively see e.g. [4] for more details. We describe the results

$$
f=6 x^{2}-6 x y-3 y^{2}-16 z^{2} .
$$

A fundamental domain is a pentagon with four right angles and one angle 0 .

We list the hyperbolic lines bounding the fundamental domain:

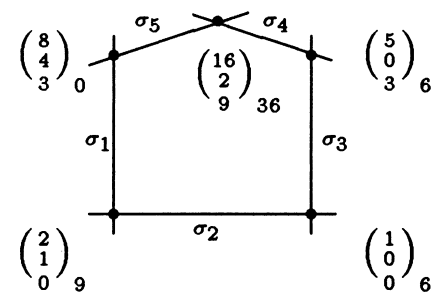

$$
\begin{aligned}
& \sigma_{1}=\left(\begin{array}{lll}
1 & 1 & 0
\end{array}\right)_{-3} \\
& \sigma_{2}=\left(\begin{array}{lll}
0 & 0 & 1
\end{array}\right)_{-16}^{-3} \\
& \sigma_{3}=(1,2,0)_{-18} \\
& \sigma_{4}=\left(\begin{array}{lll}
8 & 0 & 5
\end{array}\right)_{-16} \\
& \sigma_{5}=(6,2,3)_{-12}
\end{aligned}
$$


The reflection in the lines $\sigma_{i}$ are units. The indices denote the various spinor normes. Here are the reflections in $\sigma_{i}$ :

$$
\begin{aligned}
& X_{1}=\left(\begin{array}{ccc}
-3 & 4 & 0 \\
-2 & 3 & 0 \\
0 & 0 & -1
\end{array}\right), \quad X_{2}=\left(\begin{array}{ccc}
-1 & 0 & 0 \\
0 & -1 & 0 \\
0 & 0 & 1
\end{array}\right), \quad X_{3}=\left(\begin{array}{ccc}
-1 & 1 & 0 \\
0 & 1 & 0 \\
0 & 0 & -1
\end{array}\right) \\
& X_{4}=\left(\begin{array}{ccc}
-49 & 24 & 80 \\
0 & -1 & 0 \\
-30 & 15 & 49
\end{array}\right), \quad X_{5}=\left(\begin{array}{ccc}
-31 & 24 & 48 \\
-10 & 7 & 16 \\
-15 & 12 & 23
\end{array}\right) \\
& U=\left\langle X_{1}, X_{2}, X_{3}, X_{4}, X_{5}\right\rangle=\mathrm{SO}_{3}(f, \mathbf{Z}) \\
& g=-6 x^{2}+6 x y+3 y^{2}-16 z^{2}
\end{aligned}
$$

The fundamental domain is a pentagon with five right angles.

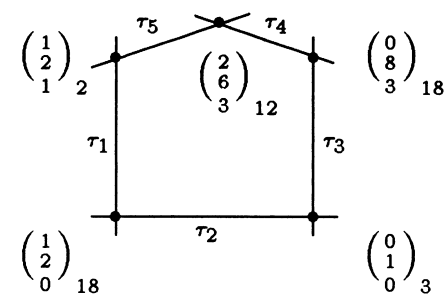

$$
\begin{aligned}
\tau_{1} & =\left(\begin{array}{lll}
1 & 0 & 0
\end{array}\right)_{-6} \\
\tau_{2} & =\left(\begin{array}{lll}
0, & 0,1
\end{array}\right)_{-16} \\
\tau_{3} & =(1,-1,0)_{-9} \\
\tau_{4} & =(0,2,1)_{-4} \\
\tau_{5} & =(8,16,9)_{-144}
\end{aligned}
$$

The reflection in the lines are units:

$$
\begin{aligned}
Y_{1}=\left(\begin{array}{ccc}
1 & -1 & 0 \\
0 & -1 & 0 \\
0 & 0 & -1
\end{array}\right), \quad Y_{2}=\left(\begin{array}{ccc}
-1 & 0 & 0 \\
0 & -1 & 0 \\
0 & 0 & 1
\end{array}\right), \quad Y_{3}=\left(\begin{array}{ccc}
1 & 0 & 0 \\
-2 & -1 & 0 \\
0 & 0 & -1
\end{array}\right) \\
Y_{4}=\left(\begin{array}{ccc}
-1 & 0 & 0 \\
-6 & -7 & 16 \\
-3 & -3 & 7
\end{array}\right), \quad Y_{5}=\left(\begin{array}{ccc}
-1 & -8 & 16 \\
0 & -17 & 32 \\
0 & -9 & 17
\end{array}\right) \\
V=\left\langle Y_{1}, Y_{2}, Y_{3}, Y_{4}, Y_{5}\right\rangle=\mathrm{SO}_{3}(g, \mathrm{Z})
\end{aligned}
$$

Let $H=\langle U, V\rangle$. We shall show that

THEOREM 3.

$$
\left|\mathrm{SL}_{3}(\mathrm{Z}): H\right|<\infty \text {. }
$$

Proof. We must try to produce sufficiently many elementary unipotent elements in $H$.

We note some computations: 


$$
\begin{aligned}
& X_{2} X_{5} X_{2} Y_{4}=\left(\begin{array}{ccc}
31 & -24 & 48 \\
16 & -1 & 0 \\
-12 & 15 & -31
\end{array}\right) \\
& \left(X_{2} X_{5} X_{2} Y_{4}\right)^{2 n}=\left(\begin{array}{ccc}
1 & 0 & 0 \\
480 n & 1-384 n & 768 n \\
240 n & -192 n & 1+384 n
\end{array}\right), n \in \mathbf{Z} \\
& W_{1}=Y_{3} \cdot\left(X_{2} X_{5} X_{2} Y_{4}\right)^{2 n} \cdot Y_{3}^{-1} \cdot\left(X_{2} X_{5} X_{2} Y_{4}\right)^{-2 n}=\left(\begin{array}{ccc}
1 & 0 & 0 \\
-1728 n & 1 & 0 \\
-864 n & 0 & 1
\end{array}\right) \\
& Y_{2} \cdot W_{1} \cdot Y_{2}^{-1}=\left(\begin{array}{ccc}
1 & 0 & 0 \\
-1728 n & 1 & 0 \\
-864 n & 0 & 1
\end{array}\right) \\
& W_{31}=Y_{2} W_{1} Y_{2}^{-1} W_{1}^{-1}=\left(\begin{array}{ccc}
1 & 0 & 0 \\
0 & 1 & 0 \\
-864 n & 0 & 1
\end{array}\right) \\
& W_{21}=W_{1}^{-2} W_{31}^{-1}=\left(\begin{array}{ccc}
1 & 0 & 0 \\
3456 n & 1 & 0 \\
0 & 0 & 1
\end{array}\right) \\
& W_{32}=\left(Y_{1} W_{31}\right)^{2}=\left(\begin{array}{ccc}
1 & 0 & 0 \\
0 & 1 & 0 \\
0 & 1728 n & 1
\end{array}\right)
\end{aligned}
$$

Take $W_{1}$ for $n=1$, obtaining

$$
\begin{gathered}
W_{2}=W_{1}^{5}\left(X_{2} X_{5} X_{2} Y_{4}\right)^{36}=\left(\begin{array}{ccc}
1 & 0 & 0 \\
0 & -6911 & 13824 \\
0 & -3456 & 6913
\end{array}\right) \\
W_{3}=Y_{1} W_{2} Y_{1}^{-1} W_{2}^{-1}=\left(\begin{array}{ccc}
1 & -6912 & 13824 \\
0 & 1 & 0 \\
0 & 0 & 1
\end{array}\right) \\
W_{12}=\left(Y_{2} W_{3}\right)^{2}=\left(\begin{array}{ccc}
1 & -13824 & 0 \\
0 & 1 & 0 \\
0 & 0 & 1
\end{array}\right)
\end{gathered}
$$




$$
\begin{aligned}
& W_{13}=Y_{2} W_{3} Y_{2}^{-1} W_{3}^{-1}=\left(\begin{array}{ccc}
1 & 0 & -27648 \\
0 & 1 & 0 \\
0 & 0 & 1
\end{array}\right) \\
& W_{23}=Y_{3} W_{13} Y_{3}^{-1} W_{13}^{-1}=\left(\begin{array}{ccc}
1 & 0 & 0 \\
0 & 1 & -55296 \\
0 & 0 & 1
\end{array}\right)
\end{aligned}
$$

Hence we have

$$
I+m e_{i j} \in H, i, j=1,2,3, i \neq j \text { for } m=55296=2^{11} \cdot 3^{3} .
$$

Using Theorem 1, we conclude

$$
\left|\mathrm{SL}_{3}(\mathrm{Z}): H\right|<\infty .
$$

It is a routine computation to produce the precise index. One has to examine the image of $\left\langle X_{1}, \ldots Y_{5}\right\rangle$ in the finite group $\mathrm{SL}_{3}\left(\mathrm{Z} / m^{2} \mathrm{Z}\right)$ for $m=2^{11} 3^{3}$.

Thanks go to the referee for useful comments and references.

It is also understood that Vaserstein's idea and its generalization such as presented in this paper lend themselves to a broad generalization, both to different types of discrete subgroups of simple Lie groups and to linear groups over more general rings where the elementary number theory used in this paper can be mimicked.

\section{REFERENCES}

1. J. Mennicke, Finite factor groups of the unimodular group, Ann. of Math. 81, No 1, 1965.

2. H. Bass, J. Milnor, J.-P. Serre, Solution of the congruence subgroup problem for $\operatorname{SL}(n)(n \geq 3)$ and $\operatorname{Sp}(2 n)(n \geq 2)$, Inst. Hautes Études Sci. Publ. Math. 33 (1967).

3. L. N. Vaserstein, On the group $\mathrm{SL}_{2}$ over Dedekind rings of arithmetical type, Mat. Sb. 89 (131): 2 (10) (1972), 313-322.

4. R. Fricke und F. Klein, Vorlesungen über die Theorie der automorphe Funktionen, Bd.1 (XIV +634 S.), 1897, Bd.2 (VIII +668 S.), Lieferung 1, 1901, Lieferung 2, 1911, Lieferung 3, 1912, Leipzig, Teubner.

5. L. N. Vaserstein, Structure of the classical arithmetic groups of rank > 1, Mat. Sb. 91 (133): 3 (7), 1973. English translation Math USSR-Sb. 20, 465-492.

6. L. N. Vaserstein, On the normal subgroups of $\mathrm{GL}_{n}$ over a ring, Lecture Notes in Math. 854 (1981), 454-465. 